\title{
Extracting Trees of Quantitative Serial Episodes`
}

\author{
Mirco Nanni ${ }^{1}$ and Christophe Rigotti ${ }^{1,2}$ \\ ${ }^{1}$ KDD Laboratory, University of Pisa and ISTI-CNR Pisa, Italy \\ ${ }^{2}$ INSA-LIRIS UMR 5205 CNRS, Lyon, France
}

\begin{abstract}
Among the family of the local patterns, episodes are commonly used when mining a single or multiple sequences of discrete events. An episode reflects a qualitative relation is-followed-by over event types, and the refinement of episodes to incorporate quantitative temporal information is still an on going research, with many application opportunities. In this paper, focusing on serial episodes, we design such a refinement called quantitative episodes and give a corresponding extraction algorithm. The three most salient features of these quantitative episodes are: (1) their ability to characterize main groups of homogeneous behaviors among the occurrences, according to the duration of the is-followedby steps, and providing quantitative bounds of these durations organized in a tree structure; (2) the possibility to extract them in a complete way; and (3) to perform such extractions at the cost of a limited overhead with respect to the extraction of standard episodes.
\end{abstract}

\section{Introduction}

Sequential data is a common form of information available in several application contexts, thus naturally inducing a strong interest for them among data analysts. A decade-long attention has been paid by researchers in data mining to study forms of patterns appropriated to this kind of data, such as sequential patterns [1] and episodes [9,7]. In particular, in this paper we will focus on serial episodes, that are sequences of event types extracted from single or multiple input sequences, and that reflect a qualitative relation is-followed-by between the event types.

Episodes have natural applications into several domains, including for instance the analysis of business time series [2], medical data [10], geophysical data [11] and also alarm log analysis for network monitoring (especially in telecommunications) [5]. However, in many applications episodes clearly show some limitations, due to the fact that the information provided by the is-followedby relation is not always enough to properly characterize the phenomena at hand. This, in particular, pulls our research toward the refinement of episodes to incorporate quantitative temporal information, able to describe the time intervals observed for the is-followed-by relation.

* This research is partly funded by EU contracts IQ IST-FP6-516169, and GeoPKDD IST-FP6-014915. 
In this paper, we propose a refinement of episodes called quantitative episodes, that provides quantitative temporal information in a readable, tree-based graphically representable form. These quantitative episodes describe the main groups of homogeneous behaviors within the occurrences of each episode, according to the elapsed times between the consecutive event types of the episode. Moreover, they are not provided in an isolated way, but in trees giving a global view of how the occurrences of the corresponding episode differentiate in homogeneous groups along the elements of the pattern. From a computational point of view, the main interest of the quantitative episodes is that they can be mined in a sound and complete way without increasing the cost of extractions significantly when compared to extractions of episodes alone. This is achieved through an extraction algorithm that tightly integrates episode extraction with a computationally reasonable analysis of temporal quantitative information.

This paper is organized as follows: in Section 2 some preliminary definitions needed concerning episodes are recalled from the literature; Section 3, then, introduces quantitative episodes; Section 4 presents the principle of an algorithm for efficiently extracting quantitative episodes, which is evaluated experimentally in Section 5; finally, in Section 6 we briefly review the related literature and conclude with a summary in Section 7 .

\section{Preliminary definitions}

We briefly introduce standard notions [8], or give equivalent definitions when more appropriated to our presentation.

Definition 1. (event, event sequence,operator $\sqsubseteq$ ) Let $E$ be a set of event types and $\prec$ a total order on $E$. An event is a pair denoted $(e, t)$ where $e \in E$ and $t \in \mathbb{N}$. The value $t$ denotes the time stamp at which the event occurs. An event sequence $S$ is a tuple of events $S=\left\langle\left(e_{1}, t_{1}\right),\left(e_{2}, t_{2}\right), \ldots,\left(e_{l}, t_{l}\right)\right\rangle$ such that $\forall i \in\{1, \ldots, l-1\}, t_{i}<t_{i+1} \vee\left(t_{i}=t_{i+1} \wedge e_{i} \prec e_{i+1}\right)$. Given two sequences of events $S$ and $S^{\prime}, S^{\prime}$ is a subsequence of $S$, denoted $S^{\prime} \sqsubseteq S$, if $S^{\prime}$ is equal to $S$ or if $S^{\prime}$ can be obtained by removing some elements in $S$.

Definition 2. (episode, occurrence, minimal occurrence, support) An episode is a non empty tuple $\alpha$ of the form $\alpha=\left\langle e_{1}, e_{2}, \ldots, e_{k}\right\rangle$ with $e_{i} \in E$ for all $i \in\{1, \ldots, k\}$. In this paper, we will use the notation $e_{1} \rightarrow e_{2} \rightarrow \ldots \rightarrow e_{k}$ to denote the episode $\left\langle e_{1}, e_{2}, \ldots, e_{k}\right\rangle$ where ' $\rightarrow$ ' may be read as 'is followed by'. The size of $\alpha$ is denoted $|\alpha|$ and is equal to the number of elements of the tuple $\alpha$, i.e., $|\alpha|=k$. The prefix of $\alpha$ is the episode $\left\langle e_{1}, e_{2}, \ldots, e_{k-1}\right\rangle$. We denote it as prefix $(\alpha)$. An episode $\alpha=\left\langle e_{1}, e_{2}, \ldots, e_{k}\right\rangle$ occurs in an event sequence $S$ if there exists at least one sequence of events $S^{\prime}=\left\langle\left(e_{1}, t_{1}\right),\left(e_{2}, t_{2}\right), \ldots,\left(e_{k}, t_{k}\right)\right\rangle$ such that $\forall i \in\{1, \ldots, k-1\}, t_{i}<t_{i+1}$ and $S^{\prime} \sqsubseteq S$. The pair $\left\langle t_{1}, t_{k}\right\rangle$ is called an occurrence of $\alpha$ in $S$. Moreover, if there is no other occurrence $\left\langle t_{1}^{\prime}, t_{k}^{\prime}\right\rangle$ such that $\left[t_{1}^{\prime}, t_{k}^{\prime}\right] \subset\left[t_{1}, t_{k}\right]$, then the pair $\left\langle t_{1}, t_{k}\right\rangle$ is called a minimal occurrence of $\alpha$. The support of $\alpha$ in $S$, denoted support $(\alpha, S)$, is the number of minimal occurrences of $\alpha$ in $S$. 
Intuitively, a minimal occurrence is simply an occurrence that does not strictly contain another occurrence of the same episode. These episodes and their occurrences correspond to the serial episodes of [8]. For instance, let $S=$ $\langle(a, 0),(b, 1),(c, 1),(b, 2)\rangle$ be an event sequence and $\alpha=a \rightarrow b$ be an episode. Then, $\alpha$ has two occurrences in $S:\langle 0,1\rangle$ and $\langle 0,2\rangle$. The former is a minimal occurrence, while the latter is not, since $[0,1] \subset[0,2]$. Notice that there is no occurrence of episode $\alpha^{\prime}=b \rightarrow c$.

These definitions, and the ones introduced in the rest of the paper, are given for a single sequence $S$, but they extend trivially to multiple sequences. In that case the support is the sum of the number of occurrences in all sequences ${ }^{1}$.

\section{Quantitative episodes}

In this section we introduce an extension of episodes that includes quantitative information. The precise definitions will be preceded by an intuitive, informal presentation of the key ideas.

\subsection{Informal presentation}

The idea of quantitative episodes essentially consists in dividing the set of occurrences of an episode into homogeneous, significantly populated groups. Homogeneity, in particular, is obtained when on each step, made of two consecutive elements of the episode, the occurrences in the same group show similar transition times (i.e., similar times elapsed between an element and the next one within the episode). The result can be graphically summarized through a treelike structure, as the one depicted in Figure 1 that represents homogeneous groups of occurrences of an episode $\alpha=A \rightarrow B \rightarrow C \rightarrow D$. The figure can be read in the following way:

- The episode has 1000 occurrences in the sequence of events, and this value is written under the first event of the episode.

- Among these 1000 occurrences, there are 2 subgroups that show homogeneous duration for step $A \rightarrow B$ : one (the upper branch of the split) corresponds to transition times between 2 and 10, and covers 500 occurrences; the other (lower branch) corresponds to transition times in interval $[15,20]$ and covers 400 occurrences. Notice that 100 occurrences of $A \rightarrow B \rightarrow C \rightarrow D$ are lost, meaning that they exhibit a rather isolated duration for step $A \rightarrow B$ and cannot be associated with other occurrences to form a significantly populated group.

\footnotetext{
${ }^{1}$ Notice that here, the support is not the number of sequences containing at least one occurrence of the pattern, as in the case of sequential patterns over a base of sequences [1].
} 
- In the largest group obtained above, all occurrences present similar step durations for steps $B \rightarrow C$ and $C \rightarrow D$, and are kept together in a single group. The other group, containing 400 occurrences, is split further into homogeneous groups w.r.t. duration of step $B \rightarrow C$. Notice that the resulting homogeneous groups overlap, sharing a subset of occurrences and resulting in non-disjoint time intervals. Indeed, we can observe that the total count of occurrences in the two groups $(205+202)$ is greater than the original total amount (400), since some occurrences are counted twice.

- One of these two groups is further split into two (disjoint) groups while the other is not.

- Each path from the root to a leaf in the tree corresponds to a group of occurrences that shows an homogeneous behavior along all the steps of the episode, and covers a sufficient number of occurrences (in this example, at least 90). This homogeneous behavior can be represented by the sequence of time intervals on the path, and can be added to the episode as a quantitative feature to form a main grouping quantitative episode. The tree in Figure 1 depicts four such patterns (one for each path from the root to a leaf). The tree relates these patterns together, showing how the occurrences can be differentiated into groups along the steps of the episode.

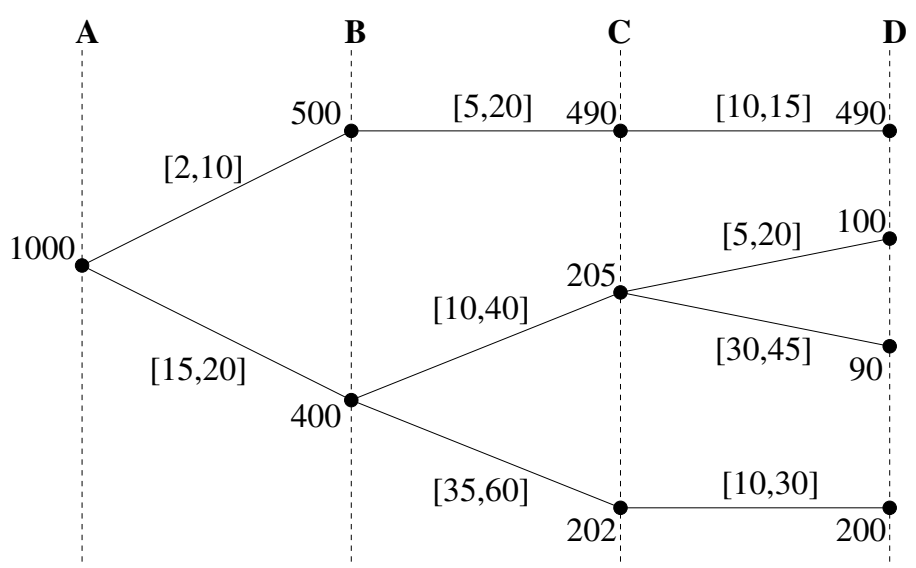

Fig. 1. Tree of quantitative episodes for episode $\alpha=A \rightarrow B \rightarrow C \rightarrow D$.

Example 1. In a medical context, we can assume to have recorded the sequences of symptoms, diseases and therapies of all patients in a hospital. Then, mining frequent quantitative episodes can yield a set of common patterns in the history of patients in terms of sequences of symptoms, etc., together with the common timings between pairs of consecutive events, that can help to characterize, e.g., the course of diseases, reactions to therapies, etc. As a fictional example, we can consider the tree of Figure 1 with $A=$ benign disease, $B=$ symptom $1, C$ 
= symptom $2, D=$ severe disease, and using days as time unit. In this case, beside the interesting evolution of a disease, the tree points out the existence of different groups of patients, each following the same evolution but with different timings. The first differentiation occurs between the appearance of the benign disease and the first symptom, in one case taking no more than 10 days, and in the other case taking approximately between two and three weeks. Then, the second group of patients further differentiate in the timings between the two symptoms (10 to 40 days in one case and 35 to 60 in the second one) and one subgroup shows differences also on the last step ( 5 to 20 days in one case and 30 to 45 in the second one). This kind of information could be useful to domain experts, both as ready-to-use knowledge for the daily care of patients, and as insight for the a more general study and understanding of the diseases involved.

Some sample quantitative episode trees, obtained on real data involving the logs of a web site, are provided in Section 5.2, Figure 10.

We notice that our approach essentially produces trees rooted on the first event of each episode, from which all the differentiations in time develop. While this choice looks very natural, since it follows the order of events in the episode, other choices could be useful in specific contexts as, for example, rooting the tree on the last event of the episode. However, such variants of the quantitative episodes studied in this paper follow the same principles, and in some cases they can be obtained by simply preprocessing the input data, e.g., moving the root of the tree on the last event of episodes can be obtained by essentially reversing the order of the input sequences.

\subsection{Quantitative episode definition}

Definition 3. (quantitative episode) A quantitative episode (q-episode) is a pair $P=\langle\alpha, I T\rangle$ where $\alpha$ is an episode of size $k>1$, and $I T=\left\langle i t_{1}, \ldots, i t_{k-1}\right\rangle$, with $\forall i \in\{1, \ldots, k-1\}, i t_{i}=\left[a_{i}, b_{i}\right] \subset \mathbb{N}^{+}$(i.e., $i t_{i}$ is an interval in $\mathbb{N}^{+}$). The size of $P$, denoted $|P|$ is defined as $|P|=|\alpha|$.

The $i t_{i}$ intervals are intended to represent values of elapsed time between the occurrences of two consecutive event types of the episode $\alpha$. For instance $\langle A \rightarrow B \rightarrow C \rightarrow D,\langle[15,20],[10,40],[5,20]\rangle\rangle$ is one of the q-episodes depicted in Figure 1.

To handle the time stamps of the events corresponding to all event types within an episode the definition of occurrence needs to be modified as follows.

Definition 4. (occurrence) An occurrence of an episode $\alpha=\left\langle e_{1}, e_{2}, \ldots, e_{k}\right\rangle$ in an event sequence $S$ is a tuple $\left\langle t_{1}, t_{2}, \ldots, t_{k}\right\rangle$ such that there exists $S^{\prime}=$ $\left\langle\left(e_{1}, t_{1}\right),\left(e_{2}, t_{2}\right), \ldots,\left(e_{k}, t_{k}\right)\right\rangle$ satisfying $\forall i \in\{1, \ldots, k-1\}, t_{i}<t_{i+1}$ and $S^{\prime} \sqsubseteq S$.

Notice that subsequence $S^{\prime}$ in the definition above can be formed by noncontiguous elements of sequence $S$.

Let us now consider the notion of minimality of occurrences. Let $S=\langle(a, 1)$, $(b, 3),(b, 6),(c, 9)\rangle$ be an event sequence and $\alpha=a \rightarrow b \rightarrow c$ be an episode. 
Then $\langle 1,3,9\rangle$ and $\langle 1,6,9\rangle$ are two occurrences of $\alpha$. If we consider a notion of minimal occurrence based only on the starting date and the ending date of the occurrences, then both are minimal. This does not fit with the intuition behind the original notion of minimal occurrence, according to which in such a situation there is only one minimal occurrence, i.e., the occurrence $\langle 1,9\rangle$ (only the starting and ending dates are used to identify occurrences in the original framework).

Thus, using occurrences that account for the intermediate time stamps as in Definition 4, the notion of minimal occurrence has to be redefined, and must not be based only on the starting and ending dates of the occurrences. Moreover, this extension has to be made by carefully avoiding counterintuitive situations. For instance, in the previous example, if we choose as minimal occurrences those containing only minimal occurrences of the different parts of the episode, then $\langle 1,3,9\rangle$ is no longer a minimal occurrence of $\alpha$, since $\langle 3,9\rangle$ is not a minimal occurrence of $b \rightarrow c$ (the minimal occurrence of this part of the episode $\alpha$ is $\langle 6,9\rangle)$. The same arises for $\langle 1,6,9\rangle$ because $\langle 1,6\rangle$ is not minimal for the part $a \rightarrow b$ (the minimal occurrence of this part of the episode $\alpha$ is $\langle 1,3\rangle$ ). Whence, $\alpha$ would have no minimal occurrence in $S$.

In the definition we retain (given as Definition 5), we use two criteria: (1) minimality based on the starting and ending dates, and (2) when several occurrences start and end at the same dates we choose the occurrence containing the earliest possible intermediate time stamps. This second condition is expressed simply through a minimality requirement with respect to the prefix of the episode. Intuitively this means that the minimal occurrence among several occurences having the same starting and ending dates is the one formed as soon as possible, e.g., in the previous example the minimal occurrence of $\alpha$ is $\langle 1,3,9\rangle$.

Definition 5. (minimal occurrence) An occurrence $\left\langle t_{1}, \ldots, t_{k}\right\rangle$ of an episode $\alpha$ in event sequence $S$ is a minimal occurrence if (1) there is no other occurrence $\left\langle t_{1}^{\prime}, \ldots, t_{k}^{\prime}\right\rangle$ of $\alpha$ such that $\left[t_{1}^{\prime}, t_{k}^{\prime}\right] \subset\left[t_{1}, t_{k}\right]$, and (2) if $k>2$ then $\left\langle t_{1}, \ldots, t_{k-1}\right\rangle$ is a minimal occurrence of $\operatorname{prefix}(\alpha)$.

As we will consider only minimal occurrences of episodes, we will simply use the term occurrence, when there is no ambiguity.

For a step $e_{i} \rightarrow e_{i+1}$ in an episode $\alpha$, and its durations among a set of occurrences of $\alpha$, now we define how these duration values are grouped. Informally, groups correspond to maximal sets of duration values that form dense intervals, where dense means that any sub-interval of significant size $w_{s}$ contains a significant number of values $n_{s}$. More precisely, $w_{s} \in \mathbb{R}, w_{s} \geq 1$ and $n_{s} \in \mathbb{N}^{+}$ are termed the density parameters and characterize the groups in the following definition.

Definition 6. (occurrence groups) Let $\mathcal{O}$ be a set of occurrences of episode $\alpha$ and $i$ be an integer parameter such that $1 \leq i<|\alpha|$ (i identifies a step $e_{i} \rightarrow e_{i+1}$ ). Let $\Delta_{i}(x)=t_{i+1}-t_{i}$ for any occurrence $x=\left\langle t_{1}, \ldots, t_{|\alpha|}\right\rangle$ (i.e., the duration of step $e_{i} \rightarrow e_{i+1}$ for occurrence $\left.x\right)$. Then, the occurrence groups of $\mathcal{O}$ at level $i$, 
denoted as $\operatorname{group}(\mathcal{O}, i)$, are defined as follows:

$$
\begin{aligned}
& \operatorname{group}(\mathcal{O}, i)=\{g \mid g \text { is a maximal subset of } \mathcal{O} \text { s.t.: } \\
& \forall a, b \in\left[\min _{x \in g} \Delta_{i}(x), \max _{x \in g} \Delta_{i}(x)\right], \\
& \left.b-a \geq w_{s} \Rightarrow\left|\left\{x \in g \mid \Delta_{i}(x) \in[a, b]\right\}\right| \geq n_{s}\right\}
\end{aligned}
$$

For example, consider the set of occurrences $\mathcal{O}=\left\{x_{1}, \ldots, x_{8}\right\}$ having the respective durations $3,4,6,6,8,9,15,16,16$ for step $e_{i} \rightarrow e_{i+1}$ (i.e., the values of $\Delta_{i}$ ). Let the density parameters be $w_{s}=3$ and $n_{s}=2$ (i.e., at least two elements in any sub-interval of size 3$)$. Then $\operatorname{group}(\mathcal{O}, i)=\left\{\left\{x_{1}, \ldots, x_{5}\right\},\left\{x_{6}, x_{7}, x_{8}\right\}\right\}$ (corresponding respectively to the durations $3,4,6,6,8,9$ and 15,16,16).

The next definition specifies the tree structure of the occurrence groups.

Definition 7. (occurrence group tree) Let $\mathcal{O}$ be the set of occurrences of episode $\alpha$. Then, the occurrence group tree (group tree for short) of $\alpha$ is a rooted tree with labelled edges such that:

- the tree has $|\alpha|$ levels, numbered from 1 (the root) to $|\alpha|$ (the deepest leaves);

- each node $v$ is associated with a set $v . g$ of occurrences of $\alpha$;

- the root is associated with root. $g=\mathcal{O}$, i.e., with all the occurrences of $\alpha$;

- if a node $v$ at level $i, 1 \leq i<|\alpha|$, is such that group $(v . g, i)=\left\{g_{1}, \ldots, g_{k}\right\}$, then it has $k$ children $v_{1}, \ldots, v_{k}$, with $v_{j} . g=g_{j}, i \in\{1, \ldots, k\}$.

- each edge connecting node $v$ at level $i$ with its child $v_{j}$ is labelled with the interval $\left[\min _{x \in v_{j} . g} \Delta_{i}(x), \max _{x \in v_{j} . g} \Delta_{i}(x)\right]$;

Notice that such tree is unique, up to permutations in the order of the children of each node. Then, the main grouping q-episodes correspond simply to the sets of occurrences that have not been separated from the root to a leaf and that have a significant size.

Definition 8. (main grouping q-episode) A q-episode $P=\langle\alpha, I T\rangle$ is said to be a main grouping q-episode if the group tree of $\alpha$ contains a path from the root to a leaf $v$ such that:

- the labels of the edges met along the path correspond to the intervals in IT;

- and $|v . g|$, called the support of $P$, is greater or equal to $\sigma_{g}$, a user defined minimum group size.

For instance, Figure 1 depicts a tree of main grouping q-episodes for $\alpha=$ $A \rightarrow B \rightarrow C \rightarrow D$ and $\sigma_{g}=90$ (a group tree restricted to paths forming main grouping q-episodes).

Since a minimal occurrence of $\alpha$ can be obtained only by extending a minimal occurrence of prefix $(\alpha)$, we have the following simple property that is used as a safe pruning criterion in the extraction principle.

Theorem 1. Let $\alpha$ be an episode such that $|\alpha|>1$. If there exists a main grouping q-episode $\langle\alpha, I T\rangle$, then there exists a main grouping q-episode $\left\langle\right.$ prefix $\left.(\alpha), I T^{\prime}\right\rangle$. 


\section{Extracting q-episodes}

In this section, we present an algorithm to extract all main grouping qepisodes, based on the computation of the group trees. Even though the notion of group tree is rather intuitive, the difficulties lay in the fact that we have to compute such a tree for every episode. We describe the overall principle of the approach and then give the corresponding abstract algorithm.

\subsection{Principle}

A simple preliminary remark is that the tree computation can be limited to episodes occurring at least $\sigma_{g}$ times, since $\sigma_{g}$ is the minimal support of a main grouping q-episode and a q-episode cannot be more frequent than its corresponding episode. However, in practice we are still facing a large number of frequent episodes. So, we propose the algorithm Q-epiMiner that interleaves frequent episode extraction and group tree computation in a tight efficient way.

Let $\alpha=\left\langle e_{1}, \ldots, e_{n}\right\rangle$ be an episode. For each event type $e_{i}$ in $\alpha, i>1$, we consider a list $D_{i}$ that collects the durations between $e_{i-1}$ and $e_{i}$, i.e., the values $\Delta_{i-1}(x)$ for all occurrences $x$ of $\alpha$, and we suppose that each $D_{i}$ is sorted by increasing duration value. By convention, for the sake of uniformity, $D_{1}$ contains a duration of 0 for all occurrences (there is no element before $e_{1}$ ).

In the following, we describe how these lists $D_{1}, \ldots, D_{n}$ can be used to compute the group tree of pattern $\alpha$, and then how they can be updated when expanding $\alpha$ with an event type $e_{n+1}$.

Splitting one node. Splitting the group of occurrences of $\alpha$ associated to one node of the tree at level $i$ (to obtain its children at level $i+1$ ) can be done simply by a single scan of the elements in the group if these elements are ordered by the duration between $e_{i}$ and $e_{i+1}$. For instance, consider a node associated to the occurrences introduced in the previous example on page 7 , corresponding to durations $[3,4,6,6,8,9,15,16,16]$, and consider the same density parameters $w_{s}=3$ and $n_{s}=2$. Then a single scan through the list allows to find the low density areas, as for example $[10,13]$ that is a sub-interval of size 3 without any element of list $[3,4,6,6,8,9,15,16,16]$ in it, and thus the scan leads to obtain the two maximal sublists satisfying the density criterion: $[3,4,6,6,8,9]$ and $[15,16,16]$. The same principle can be applied even when the maximal sublists are overlapping. For instance, if the list of durations is $[3,4,6,6,8,9,12,15,16,16]$, a single scan allows to determine that for example only one element is in interval $[10,13]$, while at least two are in the intervals (of size 3 ) $[9,12]$ and $[12,15]$. Whence we have the two maximal sublists satisfying the density criterion: $[3,4,6,6,8,9,12]$ and $[12,15,16,16]$.

In the following, we use a function named splitGroup performing this simple treatment. We suppose that it takes as input a list of occurrences in a group, sorted by duration of $e_{i} \rightarrow e_{i+1}$, and gives as output a collection of all maximal sublists satisfying the density criterion. 
Computing the whole tree. Suppose that we have already computed the groups of occurrences denoted $g_{1}, \ldots, g_{k}$ that are associated respectively to the nodes $v_{1}, \ldots, v_{k}$ of a level $i$ of the tree. These groups are split in the following way to obtain the nodes of the next level. Firstly, we create for each node $v_{j}$ an empty list denoted $v_{j}$.sortedGroup. Then we scan $D_{i+1}$ from first to last element, and for each occurrence found in $D_{i+1}$ if the occurrence is in a group $g_{j}$ then we insert the occurrence at the end of $v_{j}$.sortedGroup. Now, we have at hand for each $v_{j}$ its group of occurrences sorted by increasing duration between $e_{i}$ and $e_{i+1}$. Then, we can apply on each $v_{j}$.sortedGroup the splitGroup function to compute the children of $v_{j}$ and their associated groups of occurrences and thus obtain the next level of the group tree. Repeating this process allows to build the group tree in a levelwise way, taking advantage of the sorted lists ${ }^{2} D_{1}, \ldots, D_{n}$. In the following, we assume that such a tree is computed by a function computeTree, applied on a tuple $\left\langle D_{1}, \ldots, D_{n}\right\rangle$.

Obtaining the information needed to compute the tree. The other key operation is the efficient computation of the sorted lists $D_{1}^{\prime}, \ldots, D_{n}^{\prime}, D_{n+1}^{\prime}$ of a pattern $\alpha \rightarrow e$. Suppose that we know the list $L_{e}$ of occurrences of $\alpha \rightarrow e$, and the sorted lists $D_{1}, \ldots, D_{n}$ of durations corresponding to the occurrences of $\alpha$. Then, the main property used is that $D_{1}^{\prime}, \ldots, D_{n}^{\prime}$ are sublists of, respectively, $D_{1}, \ldots, D_{n}$, since each occurrence of $\alpha \rightarrow e$ comes from the expansion of an occurrence of $\alpha$. So a list $D_{i}^{\prime}$ can be obtained simply by scanning $D_{i}$ from the first to the last element and picking (in order) the elements in $D_{i}$ corresponding to occurrences of $\alpha$ that have been extended to form an occurrence of $\alpha \rightarrow e$. The result is a list $D_{i}^{\prime}$ sorted by increasing duration between $e_{i-1}$ and $e_{i}$. The case of the list $D_{n+1}^{\prime}$ is different since it does not correspond to durations already computed. This list is constructed by scanning $L_{e}$ to obtain the durations between $e_{n}$ and $e_{n+1}$, and then by sorting these durations in increasing order. It should be noticed that while all other operations made on lists in the algorithm are reduced to simple scans, this sort is the only operation with a non linear complexity with respect to the size of the list. Having at hand the sorted lists $D_{1}^{\prime}, \ldots, D_{n}^{\prime}, D_{n+1}^{\prime}$ we can then compute the group tree of $\alpha \rightarrow e$ by calling computeTree $\left(\left\langle D_{1}^{\prime}, \ldots, D_{n}^{\prime}, D_{n+1}^{\prime}\right\rangle\right)$.

Integration with the extraction of episodes. One remaining problem to be solved is to build the occurrence list of the episode under consideration (as the list $L_{e}$ for $\alpha \rightarrow e$ ). Fortunately, several approaches to extract episodes, or closely related patterns like sequential patterns, are based on the use of such occurrence lists (e.g., $[8,11,14])$, providing the information needed to update the duration lists $D_{i}$. The basic idea is that if we store in a list $L$ the locations (positions in the data sequence) of the occurrences of a pattern $\alpha$, then for an event type $e$, we can

\footnotetext{
${ }^{2}$ It should be noticed that the construction starts using $D_{2}$ to obtain root.sortedGroup, and that $D_{1}$ (containing only durations set to zero by convention) is not really used, but is only mentioned for the sake of the uniformity of the notation.
} 
use $^{3} L$ to build the list $L_{e}$ of occurrences of $\alpha \rightarrow e$. Notice that the expansion is made using occurrences of $e$ that are not necessarily contiguous to the last elements of the occurrences of $\alpha$. In our case, for the occurrences of an episode $\alpha=\left\langle e_{1}, \ldots, e_{n}\right\rangle$ the location information stored in $L$ are simply the time stamps of the last element $e_{n}$ of $\alpha$, sorted by increasing value. In the following, we use a function expand that takes the input sequence $S$ and $L$, and that returns a set $\mathcal{L}_{\text {exp }}$ of tuples $\left\langle e, L_{e}\right\rangle$. The set $\mathcal{L}_{\text {exp }}$ contains for each event type $e$, the list $L_{e}$ of locations of occurrences of $\alpha \rightarrow e$. As for $L$, the location information in $L_{e}$ are the time stamps of the last element of $\alpha \rightarrow e$ and $L_{e}$ is sorted by increasing location value. It should be noticed that since $L$ is ordered by occurrence time stamp, computing $\mathcal{L}_{\text {exp }}$ under the minimal occurrence semantics is linear with respect to $|S|$.

The last important aspect is the enumeration strategy of the episodes. The key remark is that a standard depth-first prefix-based strategy fits both with the episode extraction and with the use of the sorted lists $D_{i}$ to derive the sorted lists $D_{i}^{\prime}$ to compute the group trees. A depth-first approach is particularly interesting here, since it allows to limit the amount of memory needed. So, we adopt such a strategy, that can simply be sketched as follows: when an episode $\alpha$ is considered we use it as a prefix to expand it and to obtain new episodes of the form $\alpha \rightarrow e$, and then, one after the other, we consider and expand each of these $\alpha \rightarrow e$.

It should be noticed that these choices made for the part that extracts the episodes (i.e., using occurrence lists together with a depth-first strategy) correspond to a typical approach used to mine serial episodes under the minimal occurrence semantics, similar for instance to the one used in [11].

Pruning strategy and correctness. As mentioned at the beginning of the section, if an episode $\alpha$ has a support strictly less than $\sigma_{g}$ it cannot be used to form any main grouping q-episode. The same holds for any expansion of $\alpha$ since it cannot have a greater support. So, the expansion of $\alpha$ can be safely avoided. Furthermore, consider an episode $\alpha$ such that all leaves at level $|\alpha|$ are associated to groups of size strictly less than $\sigma_{g}$ ( $\alpha$ has no corresponding main grouping qepisode, but $\alpha$ itself can have a support greater or equal to $\sigma_{g}$ ). By Theorem 1 , we can also safely avoid the expansion of $\alpha$, since this expansion cannot correspond to any main grouping q-episode. The exhaustive enumeration strategy of the episodes and the safety of the pruning strategy ensure the correctness of the general extraction principle.

\subsection{Abstract algorithm}

For the sake of simplicity of the presentation we use a common data structure for all the lists $L, L_{e}, D_{i}, D_{i}^{\prime}$. Each of them is represented by a list of tuples $\langle$ occid, $t, d t\rangle$ where occid is a unique occurrence identifier, $t$ is a time of occurrence, and $d t$ is a duration between two elements in a pattern.

\footnotetext{
${ }^{3}$ Together with other information, like the data sequence itself, or the location of the occurrences of $e$.
} 


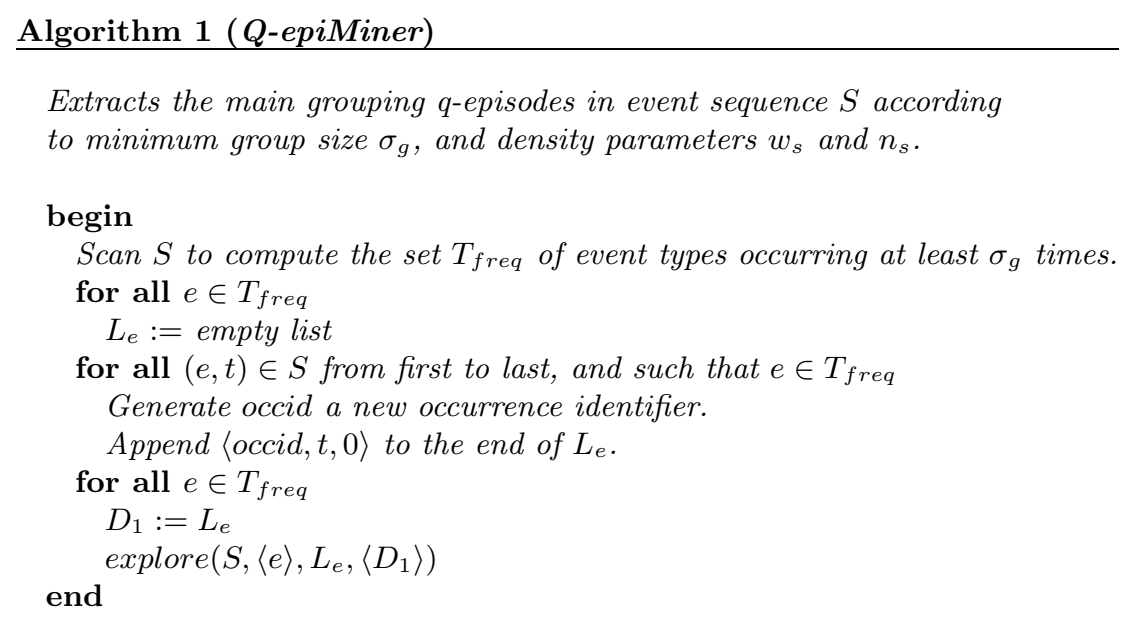

Fig. 2. Algorithm Q-epiMiner.

The extraction is performed by the Algorithm Q-epiMiner, given as Algorithm 1 (Figure 2). It first considers the patterns of size 1 and constructs the lists $L_{e}$ of occurrences of each event type $e$ (the unique occurrence identifiers are generated in any order). Then it calls explore (Algorithm 2 in Figure 3) to expand each of these patterns of size 1.

The function explore first expands the occurrences of episode $\alpha$ with respect to all event types, using expand. We required that expand preserves the occid values $^{4}$ and computes the new durations (between the two last elements of the episode). For instance, if $\langle \# 999,42,5\rangle$ is in $L$ and this occurrence can be extended by event $(\mathrm{e}, 50)$ then $\langle \# 999,50,8\rangle$ is in $L_{e}$ (where 8 is the duration, $8=50-$ 42). Next, explore takes each extension that is frequent enough (first pruning criterion), computes the lists $D_{i}^{\prime}$ from the lists $D_{i}(1<i \leq n)$ and then $D_{n+1}^{\prime}$ by sorting $L_{e}$. After having computed the group tree $T$ of the current extension (calling function computeTree), it applies the second pruning criterion and if needed makes the new pattern grow in a recursive way.

Notes on implementation. To reduce drastically the memory needed by function explore, the copy in lists $D_{1}^{\prime}, \ldots, D_{n}^{\prime}$ of the elements of lists $D_{1}, \ldots, D_{n}$ (for occid corresponding to occurrences that have been extended) is not really performed. Instead we implement a virtual deletion in the lists $D_{i}$ by hiding the elements with an occid that has not been extended (occid.isExtended $=$ false), and use these lists in place of the lists $D_{i}^{\prime}$ when calling computeTree. The hidden

\footnotetext{
${ }^{4}$ Preserving the occid is possible because under the minimal occurrence semantics, for a given event type $e$, an occurrence of $\alpha$ can be extended to form at most one occurrence of $\alpha \rightarrow e$.
} 


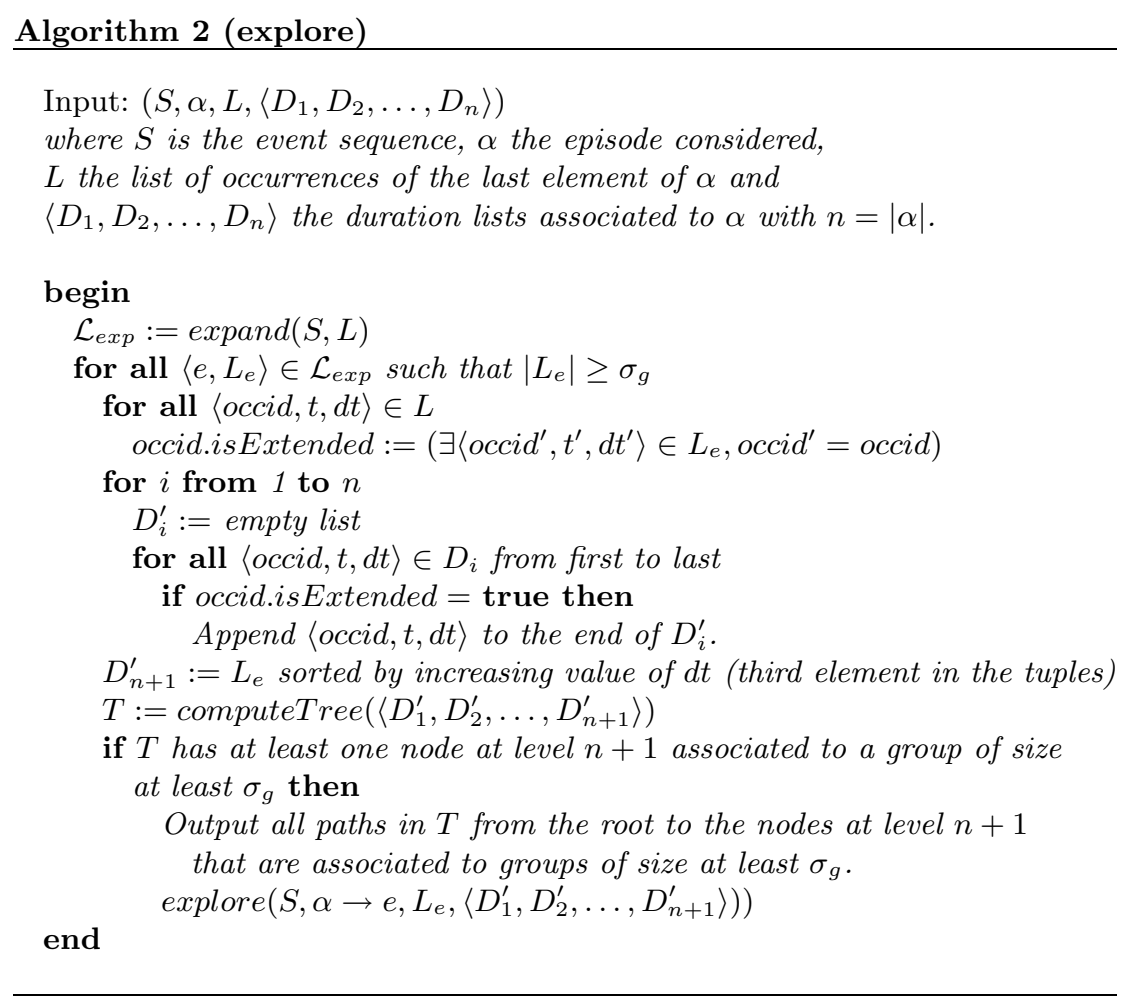

Fig. 3. Function explore.

elements are then restored in the lists $D_{i}$ before picking the next extension in $\mathcal{L}_{\text {exp }}$.

\section{Experiments}

In this section we present the results of a set of experiments, on synthetic and real datasets, mainly aimed at studying how the size of the input data and the value of some input parameters impact on the performances of the $Q$-epiMiner algorithm described in this paper. The experiments presented are made on datasets containing several sequences. As mentioned previously, the definitions extended trivially to that case (the support is simply the sum of the support in all sequences). The only change in the abstract algorithm is that the occurrence locations are not simply time stamps, but sequence identifiers together with time stamps in the sequences. The algorithm was implemented in $\mathrm{C}$, and all experiments were performed on a Intel Xeon $2 \mathrm{Ghz}$ processor with $1 \mathrm{~Gb}$ of RAM over a Linux 2.6.14 platform. 


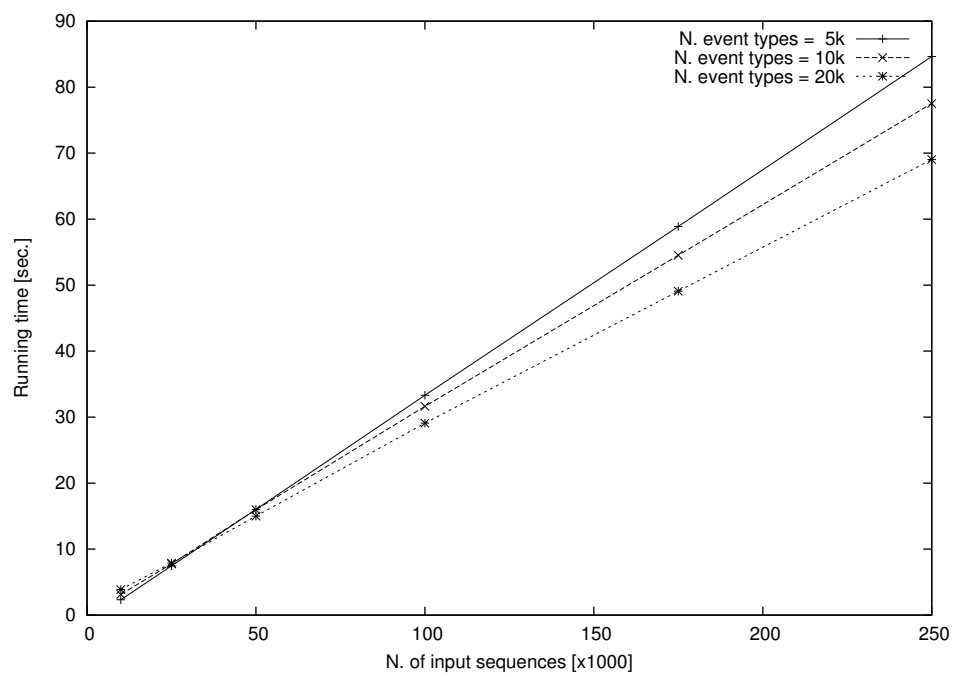

Fig. 4. Scalability w.r.t. number of input sequences.

\subsection{Performance analysis on synthetic datasets}

In order to collect large datasets having controlled characteristics, we randomly generated them by means of the Quest Synthetic Data Generator from IBM ${ }^{5}$, by varying the number of input sequences generated (from $10 \mathrm{~K}$ to $250 \mathrm{~K}$ ), the sequence length ${ }^{6}$ (from 5 to 70 ) and the number of different event types used (from $5 \mathrm{~K}$ to $20 \mathrm{~K}$ ). Where not specified hereafter, the following default parameter values were adopted: $100 \mathrm{~K}$ input sequences, sequence length equal to $25,5 \mathrm{~K}$ event types, $w_{s}=8$ and $n_{s}=4$.

The curves in Figure 4 show the execution times of the prototype over datasets of increasing size and for three different numbers of event types used in the data. The $\sigma_{g}$ parameter was set to 40 for $10 \mathrm{~K}$ sequences and then was increased proportionally, up to 1000 for $250 \mathrm{~K}$ sequences. As we can see, the execution time always grows almost linearly, having a higher slope when fewer event types are in the data ${ }^{7}$. A similar scalability analysis is provided in Figure 5, where $Q$-epiMiner is compared against the extraction of serial episodes having at least a support of $\sigma_{g}$. This extraction is performed using the frequent serial episodes mining technique embedded in Q-epiMiner, (i.e., without computing the durations, groups and trees, and implemented with the same low level optimizations). As explained in Section 4.1, this technique corresponds to a typical approach used to extract serial episodes under the minimal occurrence seman-

5 http://www.almaden.ibm.com/software/projects/iis/hdb/Projects/datamining/mining.shtml

6 The parameter of the generator controlling the number of events per time stamp was set to 1 .

${ }^{7}$ Fewer event types with the same number of sequences leads to higher supports for the remaining event types and more frequent patterns of large size. 


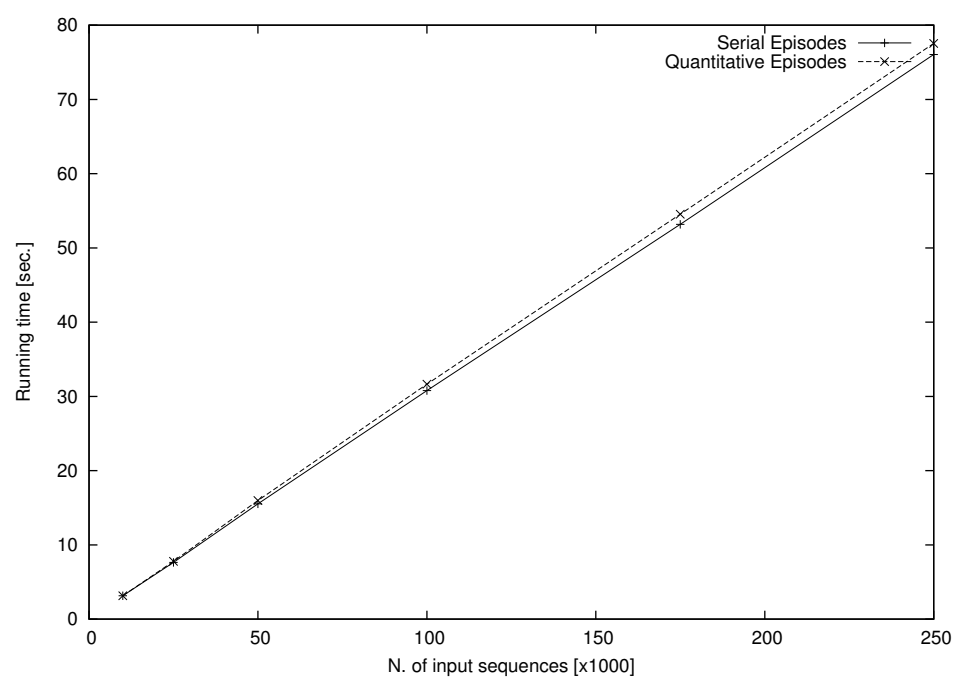

Fig. 5. Scalability comparison w.r.t. serial episode extraction.

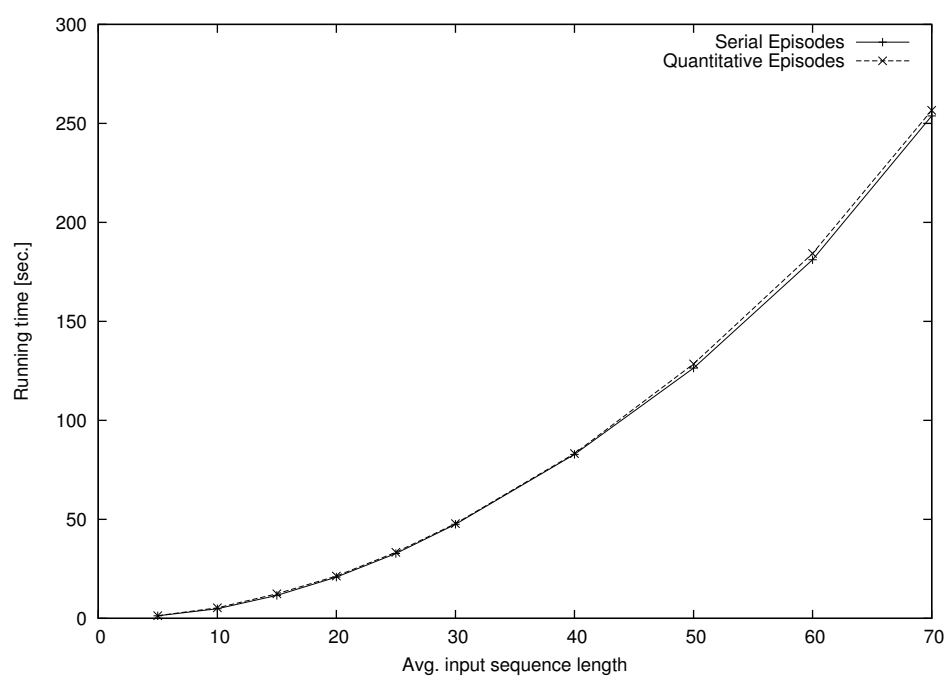

Fig. 6. Scalability w.r.t. input sequence length, with 100K sequences.

tics. The values of $\sigma_{g}$ were the same as in the previous experiment. The two curves are very close, meaning that the overhead introduced by the computation of main grouping q-episodes is well balanced by the pruning it allows. Finally, similar results are obtained by varying the length of the input sequences (see Figure 6), where both curves have an apparently-quadratic growth $\left(\sigma_{g}\right.$ was set to 80 for length 5 and then was increased proportionally, up to 1120 for length 


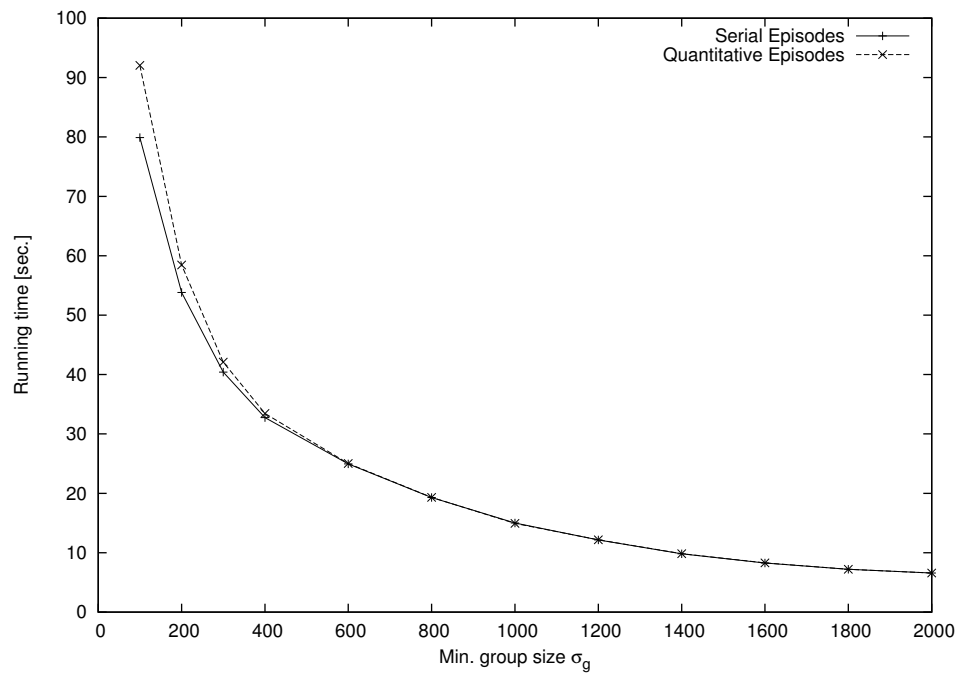

Fig. 7. Scalability w.r.t. min. group size $\sigma_{g}$, with $100 \mathrm{~K}$ sequences.

70). Obviously, for very long sequences usual episode constraints, like maximum window size, might be used [8].

Figure 7 reports the behaviour of the prototype when the minimum size of the groups is varied from 100 to 2000 , and again its comparison to the mining of frequent serial episodes at minimum support $\sigma_{g}$. Here also, the two algorithms behave very similarly, this time showing a fast drop in the execution time as $\sigma_{g}$ grows - as usual for frequent pattern mining algorithms.

\subsection{Experiments on a real dataset}

In this set of experiments we used real world data consisting of the July 2000 weblog from the web server of the Department of Electrical Engineering and Computer Sciences, University of California at Berkeley ${ }^{8}$. In a preprocessing step, all non-HTML pages where removed and user sessions were extracted, resulting in 90295 user sessions (used as input sequences) of average length of 13.0 with 72014 distinct pages.

The figure 8 describes the performances of the Q-epiMiner prototype on the Berkeley dataset for different minimum group sizes with $w_{s}=120$ (time in sec.) and $n_{s}=15$. It confirms the results obtained on synthetic data, i.e., execution times drop very quickly as $\sigma_{g}$ increases. Moreover, an additional curve is plotted that represents a version of $Q$-epiMiner that does not apply any pruning based on the absence of a main grouping q-episode, but only applies a pruning based on the support of the episodes (an episode is not expanded only when its support is strictly less than $\sigma_{g}$ ). This curve shows the effectiveness of the full pruning made

\footnotetext{
$\overline{8}$ http://www.cs.berkeley.edu/logs/http
} 


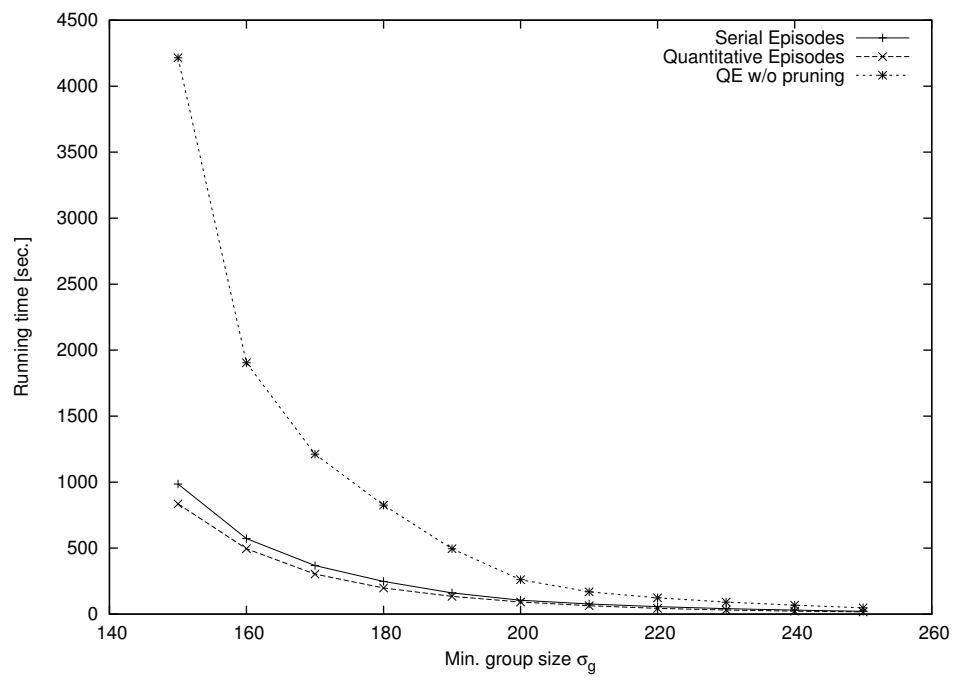

Fig. 8. Berkely dataset: Scalability w.r.t. min. group size $\sigma_{g}$.

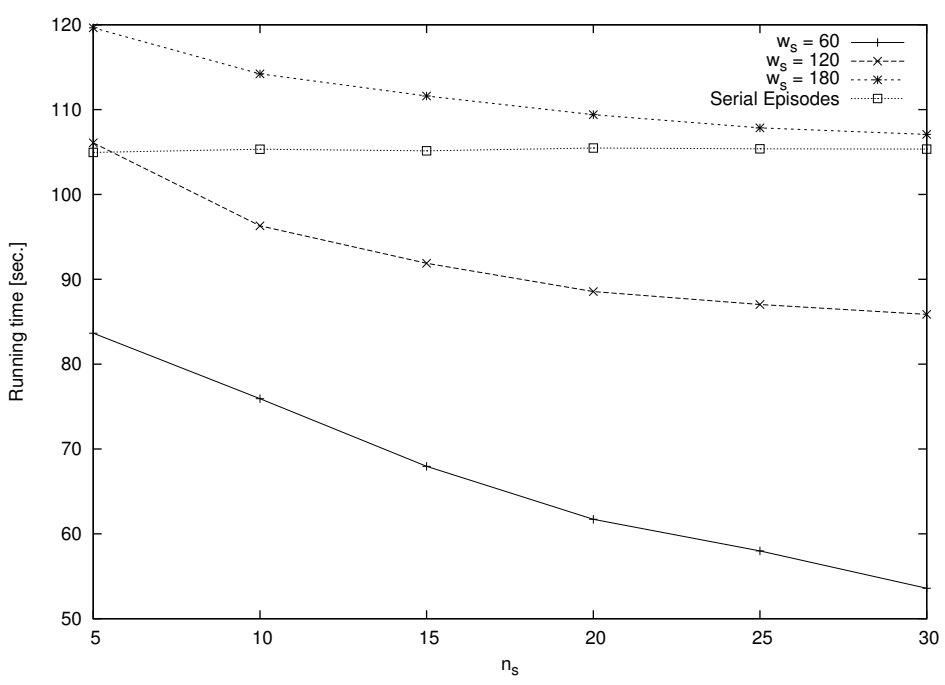

Fig. 9. Berkely dataset: effects of the density parameters.

by $Q$-epiMiner. It should also be noticed that in these experiments, Q-epiMiner performs even better than the serial episode miner (with minimum support set to $\sigma_{g}$ ), confirming the fact that the pruning capabilities of the prototype are able to balance its potential overhead.

Finally, Figure 9 presents the effect of varying the density parameters (with $\left.\sigma_{g}=200\right)$. It shows that, quite reasonably, the execution time decreases with 

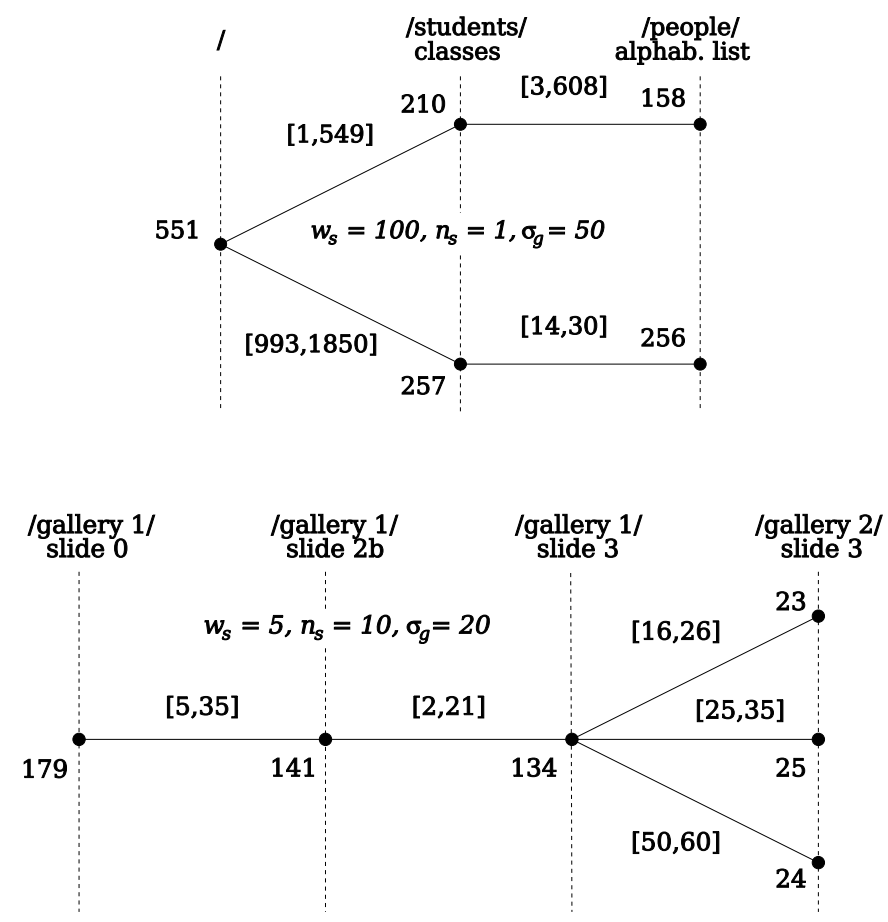

Fig. 10. Examples of trees of main grouping q-episodes.

larger minimum density parameter $n_{s}$ (since they allow a stronger pruning), and increases with larger window sizes $w_{s}$ (which acts in the opposite direction).

We conclude this section by providing in Figure 10 two sample outputs obtained from the Berkeley dataset. The first one describes a navigation pattern that starts from the web site root, visits a page about classes for students, and ends on the general alphabetically-sorted directory of people. In particular, we notice that the tree contains two groups that split at the first step, showing well separated intervals of times: $[1,549]$ against $[993,1850]$ (time in sec.). Furthermore, while the first group (which was faster in performing the first step of the episode) takes from a few seconds up to 10 minutes to move to the third page, the second group has a very compact behaviour, only taking between 14 and 30 seconds. The second output concerns the visit of a sequence of four pages in some photo galleries. The tree starts with a single branch for the first two steps, which take respectively up to 35 and up to 21 seconds, and splits only at the third step, where three groups are formed. The first two overlap ([16, 26] and $[25,35])$, therefore showing only a weak differentiation, and represent fast visitors, while the third one is separated from them, and corresponds to slow visitors that take from 50 seconds up to one minute. In both the examples, each 
time a group splits some of the occurrences it contains are lost, i.e., they are not part of any subgroup (of size at least $\sigma_{g}$ ) created by the split.

\section{Related work}

The need of quantitative temporal information in patterns over event sequences has been pointed in recent works in the data mining literature $[13,3,12$, $4,6,11]$.

An important difference between these approaches and the q-episodes introduced here, is that the former provide patterns in isolation, while q-episodes are related in tree structures. Such trees give a global view of how the occurrences of a pattern differentiate in homogeneous groups along the sequence of event types (from the first to the last element of the pattern).

Different notions of intervals are also considered. In [6] the intervals are not determined by the data but are fixed by the user; only the interval between the beginning and the end of a pattern is considered in [11]; and in [3] intervals are derived from intervals of occurrences of patterns of size two only.

The other approaches $[13,12,4]$ compute the intervals from the data and for all pattern lengths, as in the case of the q-episodes. However, among these approaches, only [4] considers an exhaustive extraction (at the cost of intrinsically expensive algorithmic solutions), while the others compute only some of the patterns using heuristics and/or non-deterministic choices.

Finally, it should be noticed that the overhead of computing the quantitative temporal information was not assessed in these previous works.

\section{Conclusion}

In this paper we introduced quantitative episodes, an extension of serial episodes that refines standard episodes by integrating quantitative temporal information. A tight integration of episode extraction and occurrence group tree computation allowed to obtain a complete and efficient algorithm that adds a negligible overhead to the extraction of serial episodes, as assessed by the experimental results on performances. These features, and the possibility of an easy-to-grasp representation of the output into a graphical tree-like structure, make the approach suitable for many applications. Future evolutions of this work will include its use in place of standard episode extraction in concrete application domains, as well as its extension to deal with quantitative aspects other than time. In particular, we aim to treat the spatial information contained in spatio-temporal sequences describing the trajectory of moving objects, such GPS traces and similar forms of data. 


\section{References}

1. R. Agrawal and R. Srikant. Mining sequential patterns. In P. S. Yu and A. S. P. Chen, editors, Proc. of the 11th International Conference on Data Engineering (ICDE'95), pages 3-14, Taipei, Taiwan, 1995. IEEE Computer Society Press.

2. G. Das, K. Lin, H. Mannila, G. Renganathan, and P. Padhraic Smyth. Rule discovery from time series. In Proc. of the 4th International Conference on Knowledge Discovery and Data Mining (KDD'98), pages 16-22, New York (USA), August 1998. AAAI Press.

3. C. Dousson and T. V. Duong. Discovering chronicles with numerical time constraints from alarm logs for monitoring dynamic systems. In Proc. of the 16th Int. Joint Conference on Artificial Intelligence (IJCAI'99), pages 620-626, San Francisco, CA, USA, 1999.

4. F. Giannotti, M. Nanni, and D. Pedreschi. Efficient mining of temporally annotated sequences. In Proc. of the SIAM Conference on Data Mining (SDM06), 2006.

5. K. Hatonen, M. Klemettinen, H. Mannila, P. Ronkainen, and H. Toivonen. TASA: Telecomunications alarm sequence analyzer or: How to enjoy faults in your network. In 1996 IEEE Network Operations and Management Symposium (NOMS'96), pages 520-529, Kyoto, Japan, April 1996.

6. Y. Hirate and H. Yamana. Sequential pattern mining with time intervals. In Proc. of the 10th Pacific-Asia Conference on Knowledge Discovery and Data Mining (PAKDD'06), 2006.

7. H. Mannila and H. Toivonen. Discovery of generalized episodes using minimal occurrences. In Proc. of the 2nd International Conference on Knowledge Discovery and Data Mining (KDD'96), pages 146-151, Portland, Oregon, August 1996.

8. H. Mannila, H. Toivonen, and A. Verkamo. Discovery of frequent episodes in event sequences. Data Mining and Knowledge Discovery, 1(3):259-298, November 1997.

9. H. Mannila, H. Toivonen, and I. Verkamo. Discovering frequent episodes in sequences. In Proc. of the 1st International Conference on Knowledge Discovery and Data Mining (KDD'95), pages 210-215, Montreal, Canada, August 1995. AAAI Press.

10. N. Meger, C. Leschi, N. Lucas, and C. Rigotti. Mining episode rules in STULONG dataset. In Proc. of the ECML/PKDD Discovery Challenge, Pisa, Italy, September 2004.

11. N. Meger and C. Rigotti. Constraint-based mining of episode rules and optimal window sizes. In Proc. of the 8th European Conf. on Principles and Practice of Knowledge Discovery in Databases (PKDD'04), pages 313-324, Pisa, Italy, September 2004. Springer-Verlag LNAI 3202.

12. A. Vautier, M.-O. Cordier, and R. Quiniou. An inductive database for mining temporal patterns in event sequences. In ECML/PKDD Workshop on Mining Spatial and Temporal Data, 2005.

13. M. Yoshida et al. Mining sequential patterns including time intervals. In Data Mining and Knowledge Discovery: Theory, Tools and Technology II (SPIE Conference), 2000.

14. M. Zaki. Spade: an efficient algorithm for mining frequent sequences. Machine Learning, Special issue on Unsupervised Learning, 42(1/2):31-60, Jan/Feb 2001. 